\title{
Antibacterial and Antibiofilm Properties of Medicinal Plant Extracts against Multi Drug Resistant Staphylococcus Species and Non Fermenter Bacteria
}

\author{
Priya Mehrishi' $^{1 *}$ (D), Priti Agarwal ${ }^{1},{\text { Shobha } \text { Broor }^{1} \text { (D) and Amisha Sharma² }}^{2}$ \\ ${ }^{1}$ Department of Microbiology, SGT Medical College, Hospital and Research Institute, SGT University, Gurugram \\ - 122 505, Haryana, India. ${ }^{2}$ Department of Microbiology, M.M.Medical College and Hospital, M.M. University \\ Solan - 173229, HP, India.
}

\begin{abstract}
Antimicrobial resistance to the pathogenic microorganism has been characterized as a public health emergency both in the community and in hospitals. That is why; we need to find alternatives, which could be used as antibacterial agents. Therefore aim of this study is to determine the antibacterial and antibiofilm properties of 4 plant extracts Clove (Syzygium aromaticum), Tea (Camellia sinensis), Garlic (Allium sativum), coriander (Coriandrum sativum). Antibacterial properties of plant extracts at different concentrations $(50,25,12.5,6.25 \mathrm{mg} / \mathrm{mL})$ were tested against Multi Drug Resistance biofilm producing Staphylococcus aureus, Pseudomonas aeruginosa, Acinetobacter baumannii, Staphylococcus epidermidis and Staphylococcus saprophyticus using the agar well diffusion method.Minimum Bactericidal Concentration (MBC) and antibiofilm properties of the plant extracts were determined using the tube dilution method and modified crystal violet assay, respectively. Total of 180 clinical isolates were screened for their MDR Pattern. Out of these, 72 were MDR isolates. These MDR isolates were categorized into weak, moderate and strong biofilm producers. Fourteen, Forty nine and nine were weak, moderate and strong biofilm producers, respectively. Out of the 4 plant extracts, Syzygium aromaticum and Camellia sinensis were found to be more effective with maximum zone of inhibition $(20-25 \mathrm{~mm}), \mathrm{MBC} 6.25 \mathrm{mg} / \mathrm{ml}$ and biofilm reduction of more than $50 \%$ compared to Allium sativum and Coriandrum sativum. All medicinal plant extracts were effective at different concentrations against the biofilm producing MDR isolates but Syzygium aromaticum and Camellia sinensis showed maximum antibacterial and antibiofilm activity.
\end{abstract}

Keywords: Antibacterial, Antibiofilm activity, Multi Drug Resistant Bacteria, Plant extracts

*Correspondence: priyamehrishi24@gmail.com; +91-9855323686

(Received: January 18, 2020; accepted: March 28, 2020)

Citation: Priya Mehrishi, Priti Agarwal, Shobha Broor and Amisha Sharma, Antibacterial and Antibiofilm Properties of Medicinal Plant Extracts against Multi Drug Resistant Staphylococcus Species and Non Fermenter Bacteria, J. Pure Appl. Microbiol., 2020; 14(1):403-413. https://doi.org/10.22207/JPAM.14.1.42

(C) The Author(s) 2020. Open Access. This article is distributed under the terms of the Creative Commons Attribution 4.0 International License which permits unrestricted use, sharing, distribution, and reproduction in any medium, provided you give appropriate credit to the original author(s) and the source, provide a link to the Creative Commons license, and indicate if changes were made. 


\section{INTRODUCTION}

Multi Drug Resistant (MDR) bacteria causing infectious diseases are a major public health problem globally. Human health, environment as well as ecosystem are equally suffering because of the excessive usage of antibiotics which result in development of multi drug resistance among pathogenic bacteria. Various reports have shown drug resistance to pathogenic bacteria ${ }^{1,2}$. Hospitals and the communities worldwide have witnessed rapid increasing of antibiotic resistance among pathogenic bacteria contributing in increased morbidity, mortality, and cost of health-care ${ }^{3,4}$. High resistance to antibiotics can be due to an important virulence factor known as bacterial biofilm which may be responsible for persistent chronic and recurrent infections. Bacterial biofilm get easily attached on to various living and nonliving solid surfaces, medical devices such as valves and catheters by forming a matrix itself $^{5}$. Therefore, the diffusion of antibiotics is hampered because of the establishment of biofilm, which results in the physiological changes in the growth mode and the low metabolic rate of inner layers of bacteria ${ }^{6,7}$. Biofilm mediated infections needs to be treated through new strategies. In this context, a renewed interest has focused on the use of medicinal plants which are natural substances, rich in secondary metabolites and are well known for their antimicrobial properties $^{8}$. Awareness about the importance of medicinal plants has been increased in the recent years despite the advances made in the field of science and research, as these medicinal plants contain certain active biological compound (phenolics,essentialoils, terpenoids, alkaloids, lectins, polypeptides, polyacetylenes) which has shown to have antibacterial properties $s^{9,10}$.

Antimicrobial resistance to the drugs used against pathogenic microorganism has been characterized as a public health emergency both in the community and hospitals. Therefore the use of Medicinal plants has been brought into consideration and is studied intensively by various researchers to know their antimicrobial activity. Researchers have also revealed the important components like eugenol in clove,catechins in tea, allicin in garlic and phytoconstituents in leaves of coriander which act as a vital source of pharmacological effects ${ }^{11,12,13,14}$. Extracts of plants contain mixtures of these components and others such as alkaloids, polyphenols and terpenoids, which are known for their antioxidant, antidiabetic, antiviral, anti-inflammatory antifungal and antimicrobial properties. So, considering the importance of medicinal plants as an antibacterial agents, current study was done to evaluate the antibacterial and antibiofilm activities of the four plants Syzygium aromaticum, Camellia sinensis, Allium sativum and Coriandrum sativum.

\section{MATERIAL AND METHODS \\ Bacterial strains}

All clinically isolated Staphylococcus aureus, Staphylococcus epidermidis, Staphylococcus saprophyticus, Pseudomonas aeruginosa, Acinetobacter baumannii from various specimens were screened for their Drug Resistance status according to CLSI guidelines - CLSI M100-S22, 2012 ${ }^{15}$. Multi drug resistant isolates were further tested for biofilm production and categorized into 3 groups - strong, moderate and weak biofilm producers ${ }^{16}$. Referral ATCC Bacterial strains of the similar isolates that have been previously characterized in Microbiology laboratory of SGT Medical College, Hospital and Research Institute, Gurugram were simultaneously tested in triplicates for antibacterial activity and single testing for biofilm inhibition assay.

\section{Collection and certification of medicinal plants}

Syzygium aromaticum - UHF herbarium no. 13632 , Camellia sinensis - 13633, Allium sativum - 13590 and Coriandrum sativum - 13634 were obtained and certified from Department of Forestry, Dr. Yashwant Singh Parmar University of Horticulture and Forestry, Nauni, Solan, Himachal Pradesh.

\section{Plant Extract Preparation}

The methanolic extracts of the above mentioned plants were prepared. Flower buds (Syzygium aromaticum), dried leaves (Camellia sinensis, Coriandrum sativum) and bulb part (Allium sativum) of plants were crushed to powder and soaked into $50 \mathrm{ml}$ of methanol. Further, it was continuously boiled for 3 minutes for 3 times, with a gap of 2 minutes interval between each boiling time. The extract or supernatant was collected, subjected to centrifugation for 5 minutes at 
$3600 \mathrm{~g}$ until clear supernatant was obtained. The supernatant was then filtered using 0.2 um filter (Micropore filters), and stored at $4{ }^{\circ} \mathrm{C}$ until further use $^{17}$.

\section{Antimicrobial activity by using Agar well diffusion method}

Sterile petri dish plates containing 20 $\mathrm{ml}$ Muller Hinton agar were prepared. Fresh culture suspensions ( 0.5 McFarland unit) of isolated pathogenic bacteria were swabbed on the respective plates. Sterile gel puncher was used to make wells over the agar plates in which plant extracts were added at various concentrations $(50,25,12.5 \& 6.25 \mathrm{mg} / \mathrm{mL})$. These plates were further incubated for 24 hours at $37^{\circ} \mathrm{C}$. After incubation,the diameter of inhibitory zones around each disc were measured in $\mathrm{mm}$ and recorded $^{17,18}$.

Determination of Minimum Bactericidal Concentration (MBC)

$M B C$ is defined as the concentration producing a $99.9 \%$ reduction in colony forming units (CFU) number in the initial inoculum. Serial two-fold dilutions of the plant extracts were made at concentration of $50,25,12.5$ and $6.25 \mathrm{mg} / \mathrm{mL}$ to which $100 \mathrm{uL}$ of microorganism suspension at a final density of $10^{5} \mathrm{cells} / \mathrm{ml}$ were added. The tubes were incubated at $37^{\circ} \mathrm{C}$ for $24 \mathrm{~h}$. The tubes after $24 \mathrm{~h}$ of incubation were sub-cultured on Mueller Hinton agar and the bacterial growth was observed on the very next day. $\mathrm{MBC}$ was determined as the lowest concentration of plant extract that failed to yield any bacterial growth in the subcultures ${ }^{19}$. Determination of Biofilm Formation by bacterial isolate using modified crystal violet assay

Sterile 96-well tissue culture plates were used to which $50 \mu$ l of Mueller-Hinton broth per well was added. Fresh bacterial suspensions (1.0 McFarland) were made and $50 \mu$ l were added to the wells and incubated for 48 hours at $37^{\circ} \mathrm{C}$. To check for the biofilm formation, contents from the wells were removed by washing with $200 \mu l$ normal saline after which $200 \mu$ l of $0.1 \%$ crystal violet stain was added and incubated again for 20 minutes. Then, each well was thoroughly washed with deionized water and later the wells were added with $200 \mu$ l of $96 \%$ ethanol. Optical density (OD) of the adherent bacteria was calculated using ELISA reader at $630 \mathrm{~nm}$. Formation of biofilm was calculated using the formula.

OD of bacteria $=[(O D$ growth control - OD sample $)$ / OD growth control] $\times 100$.

Strains were classified as follows ${ }^{20}$ : $\mathrm{OD} \leq \mathrm{ODC}=$ No biofilm producer $\mathrm{ODc}<\mathrm{OD} \leq 2 \times \mathrm{ODc}=$ Weak biofilm producer $2 \times \mathrm{ODc}<\mathrm{OD} \leq 4 \times \mathrm{ODc}=$ Moderate biofilm producer $4 \times \mathrm{ODc}<\mathrm{OD}=$ Strong biofilm producer. ODc: Optical density of growth control

\section{Determination of Anti Biofilm Activity of plant} extracts using modified crystal violet assay

Sterile 96-well tissue culture plates were used to which $50 \mu \mathrm{l}$ of Mueller-Hinton broth was added to each well. Two-fold serial dilutions of plant extract were made in the tissue culture plates. Final concentrations to be tested were 50, 25, 12.5 and $6.25 \mathrm{mg} / \mathrm{mL}$. Fresh bacterial suspensions (1.0 McFarland turbidity standard matched) were made and $50 \mu \mathrm{l}$ was added to the wells containing plant extract at different concentrations. Bacteria without plant extract was used as growth control. After 24 hrs of incubation modified crystal violet assay was performed as described above. The percentage of biofilm inhibition was calculated by using the following formula:

[(OD growth control - OD sample) / OD growth control] $\times 100$.

The biofilm inhibition concentration (BIC50) was defined as the lowest concentration of extracts that showed $50 \%$ inhibition on the biofilm formation ${ }^{20}$.

\section{RESULTS}

Out of 180 clinical isolates screened, 72 were MDR isolates. These MDR isolates were categorized into weak, moderate and strong biofilm producers.

Out of 72 MDR isolates, 14 weak, 49 moderate and 9 were strong biofilm producers. Weak biofilm producers were excluded. So total 58 biofilm producers were considered for the study as described in Table 1.

Extracts of the plants (Syzygium aromaticum, Camellia sinensis, Coriandrum sativum and Allium sativum) were tested for their antibiofilm and antimicrobial properties at 
Mehrishi et al., J. Pure Appl. Microbiol., 14(1), 403-413 | March 2020 | https://doi.org/10.22207/JPAM.14.1.42

Table 1. biofilm production by clinical MDR bacterial isolates

\begin{tabular}{lllll}
\hline Bacterial Isolates & $\begin{array}{l}\text { Number of } \\
\text { Bacteria } \\
\text { Isolates }\end{array}$ & $\begin{array}{l}\text { Strong } \\
\text { Biofilm } \\
\text { Producers }\end{array}$ & $\begin{array}{l}\text { Moderate } \\
\text { Biofilm } \\
\text { Producers }\end{array}$ & $\begin{array}{l}\text { Weak Biofilm } \\
\text { Producers }\end{array}$ \\
\hline Staphylococcus aureus & 23 & 3 & 15 & 5 \\
Pseudomonas aeruginosa & 28 & 4 & 16 & 8 \\
Acinetobacter baumannii & 8 & 2 & 5 & 1 \\
Staphylococcus epidermidis & 8 & Nil & 8 & Nil \\
Staphylococcus saprophyticus & 5 & Nil & 5 & Nil \\
\hline
\end{tabular}

Table 2. Antibacterial and antibiofilm activity of Syzygium aromaticum

\begin{tabular}{|c|c|c|c|c|}
\hline $\begin{array}{l}\text { Bacterial } \\
\text { Isolates }\end{array}$ & $\begin{array}{l}\text { centrations } \\
\mathrm{mg} / \mathrm{mL} \text { ) }\end{array}$ & $\begin{array}{c}\text { Zone of } \\
\text { Inhibition (Mean } \pm S D \text { ) }\end{array}$ & $\begin{array}{c}\mathrm{MBC} \\
(\mathrm{mg} / \mathrm{mL})\end{array}$ & $\begin{array}{l}\text { Biofilm Reduction } \\
\text { (Mean } \pm S D)\end{array}$ \\
\hline Staphylococcus & 50 & $23.6 \pm 1.20 \mathrm{~mm}$ & 6.25 & $65 \pm 0.024 \%$ \\
\hline \multirow[t]{3}{*}{ aureus (18 isolates) } & 25 & $22.2 \pm 1.38 \mathrm{~mm}$ & & $57 \pm 0.045 \%$ \\
\hline & 12.5 & $22.6 \pm 1.19 \mathrm{~mm}$ & & $54 \pm 0.027 \%$ \\
\hline & 6.25 & $20.7 \pm 1.32 \mathrm{~mm}$ & & $57 \pm 0.030 \%$ \\
\hline Staphylococcus & 50 & $23.7 \pm 0.58 \mathrm{~mm}$ & 6.25 & $63 \%$ \\
\hline \multirow[t]{3}{*}{ aureus (ATCC 25923) } & 25 & $21.0 \pm 1.00 \mathrm{~mm}$ & & $59 \%$ \\
\hline & 12.5 & $21.7 \pm 1.53 \mathrm{~mm}$ & & $55 \%$ \\
\hline & 6.25 & $20.7 \pm 1.58 \mathrm{~mm}$ & & $58 \%$ \\
\hline Pseudomonas & 50 & $23.2 \pm 1.31 \mathrm{~mm}$ & 6.25 & $54 \pm 0.020 \%$ \\
\hline \multirow{3}{*}{ aeruginosa (20 isolates) } & 25 & $24.6 \pm 1.36 \mathrm{~mm}$ & & $52 \pm 0.025 \%$ \\
\hline & 12.5 & $23.3 \pm 1.26 \mathrm{~mm}$ & & $53 \pm 0.023 \%$ \\
\hline & 6.25 & $20.0 \pm 2.28 \mathrm{~mm}$ & & $52 \pm 0.035 \%$ \\
\hline Pseudomonas & 50 & $23.3 \pm 1.53 \mathrm{~mm}$ & 6.25 & $55 \%$ \\
\hline \multirow[t]{3}{*}{ aeruginosa (ATCC 27853) } & 25 & $24.3 \pm 0.58 \mathrm{~mm}$ & & $51 \%$ \\
\hline & 12.5 & $22.3 \pm 1.15 \mathrm{~mm}$ & & $57 \%$ \\
\hline & 6.25 & $20.3 \pm 1.53 \mathrm{~mm}$ & & $54 \%$ \\
\hline Acinetobacter & 50 & $24.1 \pm 2.11 \mathrm{~mm}$ & 6.25 & $53 \pm 0.023 \%$ \\
\hline \multirow{3}{*}{ baumannii (07 isolates) } & 25 & $22.9 \pm 1.34 \mathrm{~mm}$ & & $55 \pm 2 \%$ \\
\hline & 12.5 & $22.7 \pm 1.11 \mathrm{~mm}$ & & $54 \pm 0.020 \%$ \\
\hline & 6.25 & $15.3 \pm 1.79 \mathrm{~mm}$ & & $50 \pm 0.028 \%$ \\
\hline Acinetobacter & 50 & $24.7 \pm 1.53 \mathrm{~mm}$ & 6.25 & $57 \%$ \\
\hline \multirow[t]{3}{*}{ baumannii (ATCC 19606) } & 25 & $22.7 \pm 0.58 \mathrm{~mm}$ & & $54 \%$ \\
\hline & 12.5 & $22.0 \pm 2.00 \mathrm{~mm}$ & & $53 \%$ \\
\hline & 6.25 & $16.0 \pm 1.00 \mathrm{~mm}$ & & $51 \%$ \\
\hline Staphylococcus & 50 & $21.8 \pm 1.83 \mathrm{~mm}$ & 6.25 & $65 \pm 0.027 \%$ \\
\hline \multirow{3}{*}{ epidermidis (08 isolates) } & 25 & $25.0 \pm 1.31 \mathrm{~mm}$ & & $55 \pm 0.021 \%$ \\
\hline & 12.5 & $22.6 \pm 1.41 \mathrm{~mm}$ & & $55 \pm 0.022 \%$ \\
\hline & 6.25 & $21.6 \pm 2.20 \mathrm{~mm}$ & & $50 \pm 0.032 \%$ \\
\hline Staphylococcus & 50 & $21.7 \pm 0.58 \mathrm{~mm}$ & 6.25 & $66 \%$ \\
\hline \multirow[t]{3}{*}{ epidermidis (ATCC 12228) } & 25 & $24.7 \pm 1.53 \mathrm{~mm}$ & & $57 \%$ \\
\hline & 12.5 & $23.0 \pm 1.00 \mathrm{~mm}$ & & $54 \%$ \\
\hline & 6.25 & $21.3 \pm 1.15 \mathrm{~mm}$ & & $52 \%$ \\
\hline Staphylococcus & 50 & $22.2 \pm 1.30 \mathrm{~mm}$ & 6.25 & $62 \pm 0.020 \%$ \\
\hline \multirow[t]{3}{*}{ saprophyticus (05 isolates) } & 25 & $24.8 \pm 1.30 \mathrm{~mm}$ & & $53 \pm 0.024 \%$ \\
\hline & 12.5 & $23.0 \pm 1.58 \mathrm{~mm}$ & & $52 \pm 0.018 \%$ \\
\hline & 6.25 & $21.6 \pm 1.14 \mathrm{~mm}$ & & $52 \pm 0.011 \%$ \\
\hline Staphylococcus & 50 & $23.0 \pm 1.00 \mathrm{~mm}$ & 6.25 & $65 \%$ \\
\hline \multicolumn{2}{|c|}{ saprophyticus (ATCC 15305) 25} & $23.7 \pm 1.53 \mathrm{~mm}$ & & $55 \%$ \\
\hline & 12.5 & $22.7 \pm 0.58 \mathrm{~mm}$ & & $53 \%$ \\
\hline & 6.25 & $21.0 \pm 1.00 \mathrm{~mm}$ & & $54 \%$ \\
\hline
\end{tabular}


concentration of $50,25,12.5$ and $6.25 \mathrm{mg} / \mathrm{mL}$ on 58MDR biofilm producers. Among them Syzygium aromaticum and Camellia sinensis were found to be more effective as compared to Allium sativum and Coriandrum sativum against the tested bacterial isolates.

Syzygium aromaticum showed maximum zone of inhibition $(25.0 \pm 1.31 \mathrm{~mm})$ against Staphylococcus epidermidis at $25 \mathrm{mg} / \mathrm{mL}$ concentration and minimum zone of inhibition $(15.3 \pm 1.79 \mathrm{~mm})$ against Acinetobacter baumannii at $6.25 \mathrm{mg} / \mathrm{mL}$ concentration. MBC was $6.25 \mathrm{mg} /$ $\mathrm{mL}$ for each MDR bacteria. Antibiofilm inhibition was more than $50 \%$ for all the concentrations as described in Table 2.

Camellia sinensis was effective against each bacteria at different concentrations with maximum zone of inhibition (25.4 $\pm 1.14 \mathrm{~mm})$

Table 3. Antibacterial and antibiofilm activity of Camellia sinensis

\begin{tabular}{|c|c|c|c|c|}
\hline $\begin{array}{l}\text { Bacterial } \\
\text { Isolates }\end{array}$ & $\begin{array}{l}\text { centrations } \\
\mathrm{mg} / \mathrm{mL})\end{array}$ & $\begin{array}{c}\text { Zone of } \\
\text { Inhibition (Mean } \pm S D \text { ) }\end{array}$ & $\begin{array}{c}\mathrm{MBC} \\
(\mathrm{mg} / \mathrm{mL})\end{array}$ & $\begin{array}{l}\text { Biofilm Reduction } \\
\quad(\text { Mean } \pm S D)\end{array}$ \\
\hline Staphylococcus & 50 & $25.1 \pm 1.59 \mathrm{~mm}$ & 6.25 & $73 \pm 0.023 \%$ \\
\hline \multirow[t]{3}{*}{ aureus (18 isolates) } & 25 & $23.9 \pm 1.35 \mathrm{~mm}$ & & $71 \pm 0.020 \%$ \\
\hline & 12.5 & $21.8 \pm 1.28 \mathrm{~mm}$ & & $62 \pm 0.027 \%$ \\
\hline & 6.25 & $15.0 \pm 1.30 \mathrm{~mm}$ & & $57 \pm 0.035 \%$ \\
\hline Staphylococcus & 50 & $24.6 \pm 1.15 \mathrm{~mm}$ & 6.25 & $75 \%$ \\
\hline \multirow[t]{3}{*}{ aureus (ATCC 25923) } & 25 & $22.0 \pm 1.00 \mathrm{~mm}$ & & $70 \%$ \\
\hline & 12.5 & $21.3 \pm 1.15 \mathrm{~mm}$ & & $65 \%$ \\
\hline & 6.25 & $17.6 \pm 1.53 \mathrm{~mm}$ & & $54 \%$ \\
\hline Pseudomonas & 50 & $20.3 \pm 1.49 \mathrm{~mm}$ & 6.25 & $73 \pm 0.023 \%$ \\
\hline \multirow[t]{3}{*}{ aeruginosa (20 isolates) } & 25 & $16.0 \pm 1.17 \mathrm{~mm}$ & & $71 \pm 0.032 \%$ \\
\hline & 12.5 & $15.2 \pm 1.28 \mathrm{~mm}$ & & $64 \pm 0.022 \%$ \\
\hline & 6.25 & $18.1 \pm 1.28 \mathrm{~mm}$ & & $61 \pm 0.024 \%$ \\
\hline Pseudomonas & 50 & $20.3 \pm 1.15 \mathrm{~mm}$ & 6.25 & $72 \%$ \\
\hline \multirow[t]{3}{*}{ aeruginosa (ATCC 27853) } & 25 & $17.0 \pm 1.00 \mathrm{~mm}$ & & $69 \%$ \\
\hline & 12.5 & $15.6 \pm 0.58 \mathrm{~mm}$ & & $66 \%$ \\
\hline & 6.25 & $15.3 \pm 1.53 \mathrm{~mm}$ & & $59 \%$ \\
\hline Acinetobacter & 50 & $25.1 \pm 1.46 \mathrm{~mm}$ & 6.25 & $71 \pm 0.013 \%$ \\
\hline \multirow[t]{3}{*}{ baumannii (07 isolates) } & 25 & $24.7 \pm 1.50 \mathrm{~mm}$ & & $73 \pm 0.019 \%$ \\
\hline & 12.5 & $20.1 \pm 1.35 \mathrm{~mm}$ & & $66 \pm 0.026 \%$ \\
\hline & 6.25 & $24.7 \pm 1.11 \mathrm{~mm}$ & & $63 \pm 0.021 \%$ \\
\hline Acinetobacter & 50 & $25.3 \pm 0.58 \mathrm{~mm}$ & 6.25 & $73 \%$ \\
\hline \multirow[t]{3}{*}{ baumannii (ATCC 19606) } & 25 & $24.2 \pm 1.28 \mathrm{~mm}$ & & $74 \%$ \\
\hline & 12.5 & $23.9 \pm 1.64 \mathrm{~mm}$ & & $62 \%$ \\
\hline & 6.25 & $23.7 \pm 1.16 \mathrm{~mm}$ & & $65 \%$ \\
\hline Staphylococcus & 50 & $25.3 \pm 1.19 \mathrm{~mm}$ & 6.25 & $63 \pm 0.021 \%$ \\
\hline \multirow[t]{3}{*}{ epidermidis (08 isolates) } & 25 & $24.2 \pm 1.28 \mathrm{~mm}$ & & $62 \pm 0.015 \%$ \\
\hline & 12.5 & $23.9 \pm 1.64 \mathrm{~mm}$ & & $57 \pm 0.017 \%$ \\
\hline & 6.25 & $23.7 \pm 1.16 \mathrm{~mm}$ & & $53 \pm 0.020 \%$ \\
\hline Staphylococcus & 50 & $25.3 \pm 0.58 \mathrm{~mm}$ & 6.25 & $66 \%$ \\
\hline \multirow[t]{3}{*}{ epidermidis (ATCC 12228) } & 25 & $23.7 \pm 1.50 \mathrm{~mm}$ & & $64 \%$ \\
\hline & 12.5 & $23.0 \pm 1.00 \mathrm{~mm}$ & & $54 \%$ \\
\hline & 6.25 & $20.0 \pm 1.73 \mathrm{~mm}$ & & $55 \%$ \\
\hline Staphylococcus & 50 & $25.4 \pm 1.14 \mathrm{~mm}$ & 6.25 & $75 \pm 0.020 \%$ \\
\hline \multirow[t]{3}{*}{ saprophyticus (05 isolates) } & 25 & $24.8 \pm 1.10 \mathrm{~mm}$ & & $70 \pm 2.0 \%$ \\
\hline & 12.5 & $22.0 \pm 1.58 \mathrm{~mm}$ & & $61 \pm 0.017 \%$ \\
\hline & 6.25 & $23.6 \pm 1.14 \mathrm{~mm}$ & & $60 \pm 0.015 \%$ \\
\hline Staphylococcus & 50 & $24.6 \pm 1.53 \mathrm{~mm}$ & 6.25 & $71 \%$ \\
\hline \multicolumn{2}{|c|}{ saprophyticus (ATCC 15305) 25} & $25.0 \pm 1.00 \mathrm{~mm}$ & & $73 \%$ \\
\hline & 12.5 & $22.3 \pm 1.15 \mathrm{~mm}$ & & $64 \%$ \\
\hline & 6.25 & $21.0 \pm 2.00 \mathrm{~mm}$ & & $63 \%$ \\
\hline
\end{tabular}


against Staphylococcus saprophyticus at 50mg/ $\mathrm{mL}$ concentration and minimum zone of inhibition $(15.0 \pm 1.30 \mathrm{~mm})$ against Staphylococcus aureus at $6.25 \mathrm{mg} / \mathrm{ml}$ concentration. MBC was $6.25 \mathrm{mg} /$ $\mathrm{mL}$. Antibiofilm reduction was more than $50 \%$ for each isolate at all concentrations as described in Table 3.

A. sativum did not show any antibacterial activity against $S$. epidermidis and Staphylococcus saprophyticus with no zone of inhibition but has shown maximum zone of inhibition (18. $9 \pm$ $1.61 \mathrm{~mm}$ ) against Pseudomonas aeruginosa at $50 \mathrm{mg} / \mathrm{mL}$ and minimum zone of inhibition (14.8 $\pm 1.38 \mathrm{~mm}$ ) against Staphylococcus aureus at 12.5 $\mathrm{mg} / \mathrm{mL}$ concentration. MBC came out to be 12.5 $\mathrm{mg} / \mathrm{mL}$ and reduction in biofilm formation was less than $50 \%$ (range between $20-43 \%$ ) for all concentrations as described in table 4.

Table 4. Antibacterial and antibiofilm activity of Allium sativum

\begin{tabular}{|c|c|c|c|c|}
\hline $\begin{array}{l}\text { Bacterial } \\
\text { Isolates }\end{array}$ & $\begin{array}{l}\text { centrations } \\
\mathrm{mg} / \mathrm{mL} \text { ) }\end{array}$ & $\begin{array}{c}\text { Zone of } \\
\text { Inhibition (Mean } \pm \text { SD) }\end{array}$ & $\begin{array}{c}\mathrm{MBC} \\
(\mathrm{mg} / \mathrm{mL})\end{array}$ & $\begin{array}{l}\text { Biofilm Reduction } \\
\text { (Mean } \pm S D)\end{array}$ \\
\hline Staphylococcus & 50 & $14.9 \pm 1.62 \mathrm{~mm}$ & 12.5 & $31 \pm 0.039 \%$ \\
\hline \multirow[t]{3}{*}{ aureus (18 isolates) } & 25 & $17.0 \pm 1.32 \mathrm{~mm}$ & & $22 \pm 0.025 \%$ \\
\hline & 12.5 & $14.8 \pm 1.38 \mathrm{~mm}$ & & $19 \pm 0.027 \%$ \\
\hline & 6.25 & $15.9 \pm 1.39 \mathrm{~mm}$ & & $21 \pm 0.017 \%$ \\
\hline Staphylococcus & 50 & $16.6 \pm 1.15 \mathrm{~mm}$ & 12.5 & $35 \%$ \\
\hline \multirow[t]{3}{*}{ aureus (ATCC 25923) } & 25 & $17.0 \pm 2.00 \mathrm{~mm}$ & & $25 \%$ \\
\hline & 12.5 & $16.0 \pm 1.00 \mathrm{~mm}$ & & $22 \%$ \\
\hline & 6.25 & $15.3 \pm 0.58 \mathrm{~mm}$ & & $22 \%$ \\
\hline Pseudomonas & 50 & 18. $9 \pm 1.61 \mathrm{~mm}$ & 12.5 & $32 \pm 0.022 \%$ \\
\hline \multirow[t]{3}{*}{ aeruginosa (20 isolates) } & 25 & - & & $33 \pm 0.029 \%$ \\
\hline & 12.5 & - & & $19 \pm 0.030 \%$ \\
\hline & 6.25 & - & & $20 \pm 2.0 \%$ \\
\hline Pseudomonas & 50 & $19.3 \mathrm{~mm}$ & 12.5 & $31 \%$ \\
\hline \multirow[t]{3}{*}{ aeruginosa (ATCC 27853) } & 25 & - & & $35 \%$ \\
\hline & 12.5 & - & & $24 \%$ \\
\hline & 6.25 & - & & $18 \%$ \\
\hline Acinetobacter & 50 & $14.9 \pm 1.35 \mathrm{~mm}$ & 12.5 & $25 \pm 0.052 \%$ \\
\hline \multirow[t]{3}{*}{ baumannii (07 isolates) } & 25 & $16.7 \pm 1.11 \mathrm{~mm}$ & & $22 \pm 0.025 \%$ \\
\hline & 12.5 & $15.9 \pm 1.34 \mathrm{~mm}$ & & $24 \pm 0.045 \%$ \\
\hline & 6.25 & $14.9 \pm 2.11 \mathrm{~mm}$ & & $22 \pm 0.047 \%$ \\
\hline Acinetobacter & 50 & $16.3 \pm 0.58 \mathrm{~mm}$ & 12.5 & $24 \%$ \\
\hline \multirow[t]{3}{*}{ baumannii (ATCC 19606) } & 25 & $15.3 \pm 1.20 \mathrm{~mm}$ & & $25 \%$ \\
\hline & 12.5 & $15.3 \pm 1.50 \mathrm{~mm}$ & & $23 \%$ \\
\hline & 6.25 & $13.0 \pm 1.00 \mathrm{~mm}$ & & $20 \%$ \\
\hline Staphylococcus & 50 & - & 12.5 & $34 \pm 0.086 \%$ \\
\hline \multirow[t]{3}{*}{ epidermidis (08 isolates) } & 25 & - & & $13 \pm 0.012 \%$ \\
\hline & 12.5 & - & & $36 \pm 0.101 \%$ \\
\hline & 6.25 & - & & $21 \pm 0.062 \%$ \\
\hline Staphylococcus & 50 & - & 12.5 & $37 \%$ \\
\hline \multirow[t]{3}{*}{ epidermidis (ATCC 12228) } & 25 & - & & $16 \%$ \\
\hline & 12.5 & - & & $32 \%$ \\
\hline & 6.25 & - & & $26 \%$ \\
\hline Staphylococcus & 50 & - & 12.5 & $43 \pm 0.028 \%$ \\
\hline \multirow[t]{3}{*}{ saprophyticus (05 isolates) } & 25 & - & & $41 \pm 0.064 \%$ \\
\hline & 12.5 & - & & $36 \pm 0.030 \%$ \\
\hline & 6.25 & - & & $20 \pm 0.105 \%$ \\
\hline Staphylococcus & 50 & - & 12.5 & $47 \%$ \\
\hline \multicolumn{2}{|c|}{ saprophyticus (ATCC 15305) 25} & - & & $44 \%$ \\
\hline & 12.5 & - & & $33 \%$ \\
\hline & 6.25 & - & & $24 \%$ \\
\hline
\end{tabular}


Coriandrum sativum has shown maximum zone of inhibition $(17.4 \pm 1.27 \mathrm{~mm})$ against Acinetobacter baumannii at $50 \mathrm{mg} / \mathrm{mL}$ concentration and minimum zone of inhibition $(9.80 \pm 1.25 \mathrm{~mm})$ against Staphylococcus epidermidis at $6.25 \mathrm{mg} / \mathrm{mL}$ concentration. $\mathrm{MBC}$ was varying for each bacterial isolate ranging from 12.5-50 mg/mL. Biofilm reduction of all bacteria were less than $50 \%$ for all extract concentrations as described in table 5 .

Table 5. Antibacterial and antibiofilm activity of Coriandrum sativum

\begin{tabular}{|c|c|c|c|c|}
\hline $\begin{array}{l}\text { Bacterial } \\
\text { Isolates }\end{array}$ & $\begin{array}{l}\text { centrations } \\
\mathrm{mg} / \mathrm{mL} \text { ) }\end{array}$ & $\begin{array}{c}\text { Zone of } \\
\text { Inhibition (Mean } \pm S D \text { ) }\end{array}$ & $\begin{array}{c}\mathrm{MBC} \\
(\mathrm{mg} / \mathrm{mL})\end{array}$ & $\begin{array}{l}\text { Biofilm Reduction } \\
\quad(\text { Mean } \pm \text { SD) }\end{array}$ \\
\hline Staphylococcus & 50 & $15.9 \pm 1.55 \mathrm{~mm}$ & 12.5 & $42 \pm 0.066 \%$ \\
\hline \multirow[t]{3}{*}{ aureus (18 isolates) } & 25 & $15.6 \pm 1.50 \mathrm{~mm}$ & & $44 \pm 0.027 \%$ \\
\hline & 12.5 & $14.6 \pm 1.14 \mathrm{~mm}$ & & $36 \pm 0.103 \%$ \\
\hline & 6.25 & $15.7 \pm 1.32 \mathrm{~mm}$ & & $38 \pm 0.048 \%$ \\
\hline Staphylococcus & 50 & $17.0 \pm 1.00 \mathrm{~mm}$ & 12.5 & $45 \%$ \\
\hline \multirow[t]{3}{*}{ aureus (ATCC 25923) } & 25 & $16.3 \pm 0.58 \mathrm{~mm}$ & & $46 \%$ \\
\hline & 12.5 & $14.6 \pm 0.58 \mathrm{~mm}$ & & $38 \%$ \\
\hline & 6.25 & $13.3 \pm 1.53 \mathrm{~mm}$ & & $35 \%$ \\
\hline Pseudomonas & 50 & 14. $8 \pm 1.71 \mathrm{~mm}$ & 25.0 & $42 \pm 0.040 \%$ \\
\hline \multirow[t]{3}{*}{ aeruginosa (20 isolates) } & 25 & 12. $2 \pm 1.86 \mathrm{~mm}$ & & $39 \pm 0.115 \%$ \\
\hline & 12.5 & $14.6 \pm 1.69 \mathrm{~mm}$ & & $39 \pm 0.058 \%$ \\
\hline & 6.25 & $11.9 \pm 1.37 \mathrm{~mm}$ & & $27 \pm 0.106 \%$ \\
\hline Pseudomonas & 50 & $15.3 \pm 0.58 \mathrm{~mm}$ & 25.0 & $40 \%$ \\
\hline \multirow[t]{3}{*}{ aeruginosa (ATCC 27853) } & 25 & $13.0 \pm 1.00 \mathrm{~mm}$ & & $41 \%$ \\
\hline & 12.5 & $13.6 \pm 1.53 \mathrm{~mm}$ & & $37 \%$ \\
\hline & 6.25 & $10.6 \pm 1.53 \mathrm{~mm}$ & & $30 \%$ \\
\hline Acinetobacter & 50 & $17.4 \pm 1.27 \mathrm{~mm}$ & 25.0 & $42 \pm 0.040 \%$ \\
\hline \multirow[t]{3}{*}{ baumannii (07 isolates) } & 25 & $15.6 \pm 1.51 \mathrm{~mm}$ & & $39 \pm 0.115 \%$ \\
\hline & 12.5 & $16.7 \pm 1.49 \mathrm{~mm}$ & & $39 \pm 0.058 \%$ \\
\hline & 6.25 & $16.8 \pm 1.46 \mathrm{~mm}$ & & $27 \pm 0.106 \%$ \\
\hline Acinetobacter & 50 & $17.6 \pm 0.58 \mathrm{~mm}$ & 25.0 & $39 \%$ \\
\hline \multirow[t]{3}{*}{ baumannii (ATCC 19606) } & 25 & $16.0 \pm 1.00 \mathrm{~mm}$ & & $41 \%$ \\
\hline & 12.5 & $15.3 \pm 1.15 \mathrm{~mm}$ & & $43 \%$ \\
\hline & 6.25 & $14.6 \pm 1.53 \mathrm{~mm}$ & & $31 \%$ \\
\hline Staphylococcus & 50 & $14.8 \pm 1.49 \mathrm{~mm}$ & 50.0 & $34 \pm 0.086 \%$ \\
\hline \multirow[t]{3}{*}{ epidermidis (08 isolates) } & 25 & $11.1 \pm 1.46 \mathrm{~mm}$ & & $13 \pm 0.012 \%$ \\
\hline & 12.5 & $13.0 \pm 1.31 \mathrm{~mm}$ & & $36 \pm 0.101 \%$ \\
\hline & 6.25 & $9.80 \pm 1.25 \mathrm{~mm}$ & & $21 \pm 0.062 \%$ \\
\hline Staphylococcus & 50 & $14.6 \pm 0.58 \mathrm{~mm}$ & 50.0 & $37 \%$ \\
\hline \multirow[t]{3}{*}{ epidermidis (ATCC 12228) } & 25 & $12.6 \pm 0.58 \mathrm{~mm}$ & & $16 \%$ \\
\hline & 12.5 & $13.0 \pm 1.00 \mathrm{~mm}$ & & $33 \%$ \\
\hline & 6.25 & $10.6 \pm 1.53 \mathrm{~mm}$ & & $24 \%$ \\
\hline Staphylococcus & 50 & $15.2 \pm 1.48 \mathrm{~mm}$ & 50.0 & $43 \pm 0.028 \%$ \\
\hline \multirow[t]{3}{*}{ saprophyticus (05 isolates) } & 25 & $13.2 \pm 1.30 \mathrm{~mm}$ & & $41 \pm 0.064 \%$ \\
\hline & 12.5 & $13.0 \pm 1.48 \mathrm{~mm}$ & & $36 \pm 0.030 \%$ \\
\hline & 6.25 & $12.8 \pm 1.64 \mathrm{~mm}$ & & $20 \pm 0.105 \%$ \\
\hline Staphylococcus & 50 & $15.3 \pm 1.53 \mathrm{~mm}$ & 50.0 & $45 \%$ \\
\hline \multirow[t]{3}{*}{ saprophyticus (ATCC 15305} & 5) 25 & $13.6 \pm 0.58 \mathrm{~mm}$ & & $44 \%$ \\
\hline & 12.5 & $12.00 \pm 1.00 \mathrm{~m}$ & & $34 \%$ \\
\hline & 6.25 & $10.3 \pm 1.53 \mathrm{~mm}$ & & $26 \%$ \\
\hline
\end{tabular}

\section{DISCUSSION}

This study was aimed to detect antibacterial and antibiofilm properties of four medicinal plant extracts. As we know, multi drug resistance against most commonly used chemical drugs is a highly faced problem nowadays and it is a matter of concern. Therefore, our area of interest is more focused on natural products that can be 
used as an alternative to the antimicrobials.

Syzygium aromaticum has shown maximum zone of inhibition at $50 \mathrm{mg} / \mathrm{mL}$ concentration against Staphylococcus aureus (23.6 $\pm 1.20 \mathrm{~mm})$, Pseudomonas aeruginosa (24.6 $\pm 1.36 \mathrm{~mm})$, Acinetobacter baumannii (24.1 $\pm 2.11 \mathrm{~mm}$ ), Staphylococcus epidermidis (25 \pm $1.31 \mathrm{~mm}$ ) and Staphylococcus saprophyticus (24.8 $\pm 1.3 \mathrm{~mm}$ ) at 50 and $25 \mathrm{mg} / \mathrm{mL}$ concentration. Similar results were shown by Anita et al. ${ }^{21}$ who revealed inhibition zone of $28 \mathrm{~mm}$ for Staphylococcus aureus and $30 \mathrm{~mm}$ for Pseudomonas aeruginosa at $25 \mathrm{mg} /$ $\mathrm{ml}$ concentration. Another Study done by Neelima et al., has also shown almost similar results where zone of inhibition by Syzygium aromaticum against Pseudomonas aeruginosa was between $15-30 \mathrm{~mm}$ at different concentrations $(25,50,100,200 \mu \mathrm{g} /$ $\mathrm{ml})^{22}$. Liaqat et al. ${ }^{23}$ has reported $\mathrm{MBC}$ value $(20 \mathrm{mg} / \mathrm{mL})$ of clove against $E$. coli whereas another study done by Mahajan et al. ${ }^{24}$ has shown MBC against Staphylococcus aureus and Pseudomonas aeruginosa ranging between $6.25-25 \mathrm{mg} / \mathrm{ml}$, which is slightly higher to our study in which $\mathrm{MBC}$ came out to be $6.25 \mathrm{mg} / \mathrm{mL}$ against all biofilm producing MDR isolates. In the present study, biofilm reduction was more than $50 \%$ at each concentration of clove extracts $(50,25,12.5,6.25$ $\mathrm{mg} / \mathrm{mL}$ ). Significant biofilm reduction by clove at different concentrations has also been reported by other authors too 25,26 . These results show that the methanolic extract of clove is effective against biofilm producing MDR isolates.

C. sinensis also proved its antibacterial and antibiofilm activity at all studied concentrations. It has shown highest zone of inhibition (25.1 $\pm 1.59 \mathrm{~mm}$ ) at $50 \mathrm{mg} / \mathrm{ml}$ concentration against Staphylococcus aureus, Staphylococcus epidermidis (25.3 $\pm 1.19 \mathrm{~mm})$ Staphylococcus saprophyticus (25.4 $\pm 1.14 \mathrm{~mm})$, Acinetobacter baumannii (25.1 \pm 1.46 ) and Pseudomonas aeruginosa (20.3 \pm $1.49 \mathrm{~mm})$. Compared to our study, Mehta et al. ${ }^{12}$ has shown highest zone of inhibition $(15 \mathrm{~mm})$ at $50 \mathrm{mg} / \mathrm{ml}$ against MDR Pseudomonas aeruginosa and E. coli, and $10 \mathrm{~mm}$ against Staphylococcus aureus. Another study by Archana et al. ${ }^{27}$ has shown zone of inhibition of $16 \mathrm{~mm}, 12 \mathrm{~mm}$ against Staphylococcus aureus and Pseudomonas aeruginosa respectively at different concentrations ranging from $20-100 \mu l$. These results were in concordance to our study. Liaqat et al. ${ }^{26}$ has also shown in his study that methanolic extract of Camellia sinensis was effective in reducing the biofilm formation at concentrations from $5-45 \mathrm{mg} /$ $\mathrm{ml}$ and their range of $\mathrm{MBC}$ was $20-40 \mathrm{mg} / \mathrm{ml}$ against MDR isolates whereas in our study the $\mathrm{MBC}$ value of Camellia sinensis was $6.25 \mathrm{mg} / \mathrm{ml}$ and biofilm inhibition was significantly more than $50 \%$ for all concentrations. Study done by Fakheri ${ }^{28}$ also gave $\mathrm{MBC}$ value of $2.5 \mathrm{mg} / \mathrm{ml}$ against Staphylococcus aureus and $1.25 \mathrm{mg} / \mathrm{ml}$ for Staphylococcus saprophyticus which is quite similar to our study. This proves that Camellia sinensis does possess antibacterial property. Bacterial susceptibility to Camellia sinensis extract is because of the known bactericidal effect of epigallocatechin-gallate (polyphenolic fractions of catechin component of Camellia sinensis) which is attributed to membrane perturbation ${ }^{12}$.

Allium sativum has shown decent results with zone of inhibitions between $18.9 \pm 1.61$ to $14.8 \pm 1.38 \mathrm{~mm}$ against Pseudomonas aeruginosa, Staphylococcus aureus and Acinetobacter baumannii at different concentrations. It did not show any activity against Staphylococcus epidermidis and Staphylococcus saprophyticus. Other study done by Mohsenipou et al. ${ }^{29}$ has shown no zone of inhibition by Allium sativum extract except against Bacillus cereus $(8 \mathrm{~mm})$ whereas Lekshmi et al. ${ }^{30}$ has mentioned the zone diameter of $13.8 \pm 0.29 \mathrm{~mm}$ against Staphylococcus aureus which relates to our study. In the present study, Biofilm inhibition was less than 50\% (20$30 \%$ ) at each concentration whereas Lekshmi et al., has mentioned more than $50 \%$ biofilm inhibition in which in contrast to the present study. Another study by Shams et al., has shown the concordant results to the study as reduction in biofilm was moderate which is less than $50 \%{ }^{31}$. Mohsenipou et al., have shown the $\mathrm{MBC}$ ranged between 2.5-5.0 $\mathrm{mg} / \mathrm{ml}$ against Staphylococcus aureus and Pseudomonas aeruginosa which is comparatively lower to our study where $\mathrm{MBC}$ was $12.5 \mathrm{mg} / \mathrm{ml}^{29}$. Al-Bayati stated that presence of higher content of organo-sulphur compounds and thiosulfate compound (Allicin) in the Allium sativum are responsible for its antibacterial effects. Variation in the inhibitory zone in different bacterial isolates are may be due to permeability of allicin and other components of Allium sativum to the bacteria ${ }^{32}$. 
Coriandrum sativum has shown maximum antibacterial activity against Acinetobacter baumannii with zone of inhibition $17.4 \pm$ 1.27 to $15.6 \pm 1.51 \mathrm{~mm}$ and minimum against Staphylococcus epidermidis with zone of inhibition $14.8 \pm 1.49$ to $9.8 \pm 1.25 \mathrm{~mm}$ at all concentrations. Study done by Rathabai has shown comparatively less zone of inhibition of $9.90 \pm 0.10 \mathrm{~mm}$ and $12.17 \pm 0.29 \mathrm{~mm}$ against $P$ seudomonas aeruginosa and Staphylococcus aureus by methanolic extract of Coriandrum at higher concentration of $(1 \mathrm{gm} /$ $\mathrm{ml})^{33}$. Another study done by Bakhet et al. showed that the extract of $C$. sativum when used in different concentrations (100, 50 and $10 \%$ ) has shown inhibition zones of $13-11 \mathrm{~mm}$ for Staphylococcus aureus, $13 \mathrm{~mm}$ for E. coli, 9-7mm for Pseudomonas aeruginosa which is quite close to our study ${ }^{34}$. $M B C$ in our study was found to be $12.5 \mathrm{mg} / \mathrm{ml}$ for Staphylococcus aureus but for Acinetobacter baumanni and Pseudomonas aeruginosa, the $M B C$ was $25 \mathrm{mg} / \mathrm{ml}$. Similar findings were shown by Alireza et al. ${ }^{35}$ who reported the MBC value of $25 \mathrm{mg} / \mathrm{ml}$ against Staphylococcus aureus and $50 \mathrm{mg} / \mathrm{mL}$ for Pseudomonas aeruginosa. Our study has showed that Coriandrum sativum did not have much effect on inhibiting the biofilm and it was supported by study done by Bezalwar et $a . .^{36}$ and Abraham et $a l .{ }^{37}$ as their research revealed no effect of coriander extract on biofilm inhibition. These results showed that the Coriandrum sativum was not efficient in reducing biofilm but have certain antibacterial properties when used at higher concentration range. The difference in the antimicrobial properties of these herbs to the bacterial strains is may be due to different bio-reactive substances present in extracts with different processing techniques.

\section{CONCLUSION}

In this study, all the four plant extracts have shown their effectiveness against the multidrug-resistant bacteria but overall Syzygium aromaticum and Camellia sinensis were found to be better than Allium sativum and Coriandrum sativum.

\section{ACKNOWLEDGMENTS}

The authors acknowledged Department of Microbiology, SGT Medical College, Hospital and
Research Institute for providing the research facility.

\section{CONFLICT OF INTEREST}

All authors declare that there is no conflict of interest.

\section{AUTHORS' CONTRIBUTION}

All authors listed above have made a substantial, direct and intellectual contribution to the work and approved it for publication.

\section{FUNDING}

None.

\section{DATA AVAILABILITY}

All datasets analyzed during this study are included in the manuscript.

\section{ETHICS STATEMENT}

This article does not contain any study with human participants or animals performed by any Authors.

\section{REFERENCES}

1. Faujdar SS, Bisht D, Sharma A. Antibacterial activity of Syzygium aromaticum (clove) against uropathogens producing ESBL, MBL, and AmpC beta-lactamase: Are we close to getting a new antibacterial agent? J Family Med Prim Care. 2020; 9(1):180-186. PMID: 32110587; PMCID: PMC7014883. https://doi.org/10.4103/jfmpc. jfmpc_908_19

2. Nitisha Malik, DakshinaBisht and Sameer Singh Faujdar. Extended Spectrum $\beta$ Lactamases and Metallo $\beta$-Lactamases Production in Klebsiella pneumoniae Isolates Causing Pneumonia in Rural Population of Uttar Pradesh. Int.J.Curr.Microbiol.App.Sci. 2019; 8(06): 1732- 1738. https://doi.org/10.20546/ ijcmas.2019.806.207

3. Jastaniah SD. The antimicrobial activity of some plant extracts, commonly used by Saudi people, against multidrug resistant bacteria. Life Sci J. 2014; 11(8):78-84.

4. Mill Robertson FC, Onyeka Cl, Tay SCK, Walana W. In vitro antimicrobial activity of antibact, and herbal medicinal productt against standard and clonicalbacterila isolates. J Med Plants Res. 2015; 9(11): 370-8. https://doi.org/10.5897/JMPR2015.5758

5. S.S. Grant and D.T. Hung. Persistent bacterial infections, antibiotic tolerance, and the oxidative stress response. Virulence 2013; 4(4): 273-283. https://doi. org/10.4161/viru.23987

6. T.B. Rasmussen and M. Givskov.Quorum-sensing inhibitors as anti-pathogenic drugs. International J Med Microbiol., 2006; 296(2-3), 149-161. https://doi. 
org/10.1016/j.ijmm.2006.02.005

7. Sun F, Qu F, Ling Y, Mao P, Xia P, Chen H, et al. Biofilmassociated infections: antibiotic resistance and nove therapeutic strategies. Future Microbiology 2013; 8(7): 877-886. https://doi.org/10.2217/fmb.13.58

$8 . \quad$ L. C. Simoes, M. Simoes, andM. J. Vieira. Biofilm interactions between distinct bacterial genera isolated from drinking water. Applied and Environmental Microbiology 2007; 73(19): 6192-6200. https://doi. org/10.1128/AEM.00837-07

9. Mickiene R, Bakutis B, Baliukoniene V. Antimicrobial activity of two essential oils. Ann Agric Environ Med 2011; 18: 139-144.

10. Prashant KR, Dolly J, Singh KR, Gupta KR, Watal G. Glycemic properties of Trichosanthes dioica leaves. Pharm Biol 2008; 46(12): 894-899. https://doi. org $/ 10.1080 / 13880200802370167$

11. Nagwa A. Abdallah, Rafat Z. Abd El-Rahman, Arega K. Amer, Lamia I. M. Ismaeel. Antibacterial Activity of Some Plant Extracts on The Treatment of Surgical Wounds Infection. Egypt. J. Exp. Biol. (Bot.) 2013; 9(1): $115-124$

12. Abhishek Mehta, Gaurav Saxena and Abin Mani. Comparative Analysis of Antibacterial Activity of Aqueous, Ethanolic, Methanolic and Acetone Extracts of Commercial Green Tea and Black Tea against Standard Bacterial Strains. Int.J.Curr.Microbiol.App. Sci. 2016;5(11): 145-152. https://doi.org/10.20546/ ijcmas.2016.511.017

13. Ninyio, Nathaniel Nyakaat Fadason, Tayaza Bala and Maidawa, Godwin Zakariya. Anti-Biofilm Effect of Allium sativum Extract on Clinical Isolates of Staphylococcus aureus. Nigerian Journal of Microbiology 2016; 30(2): 3494-3500.

14. Ankita Verma, Deepali Agarwal, Jyotsna Dhanik, Neelam Arya and Vivekanand. Antibacterial Efficacy and Cluster Analysis of Genotypic Extracts of Coriandrum Leaves and Seeds against Human Pathogenic Bacterial Strains. Int.J.Curr.Microbiol.App.Sci. 2018; 7(05): 2727-2736. https://doi.org/10.20546/ijcmas.2018.705.316

15. Magiorakos AP, Srinivasan A, Carey RB, Carmeli Y, Falagas ME, Giske CG, et al. Multidrug-resistant, extensively drug-resistant and pandrug-resistant bacteria: an international expert proposal for interim standard definitions for acquired resistance. Clin Microbiol Infect. 2012; 18: 268-81. https://doi. org/10.1111/j.1469-0691.2011.03570.x

16. O'toole, G.A., Kolter, R. Initiation of biofilm formation in Pseudomonas fluorescens WCS365 proceeds via multiple, convergent signalling pathways: a genetic analysis. Mol. Microbiol 1998; 28, 449-461. https:// doi.org/10.1046/j.1365-2958.1998.00797.x

17. Syed H. Abidi, Khalid Ahmed, Sikander K. Sherwani and Shahana U. Kazm. Reduction and removal of Pseudomonas aeruginosa biofilm by naturalagents. International Journal of Chemical and Pharmaceutical Sciences 2014; 5(1): 28-34.

18. Shakti Rath, Debasmita Dubey, Mahesh C Sahu, Nagen K Debata, Rabindra N Padhy. Surveillance of multidrug resistance of 6 uropathogens in a teaching hospital and in vitro control by 25 ethnomedicinal plants used by an aborigine of India. Asian Pacific Journal of Tropical Biomedicine 2012; S818-S829. https://doi. org/10.1016/S2221-1691(12)60319-0

19. JD N'guessan, M R Dinzedi, N Guessennd, A Coulibaly, M Dosso, A J Djaman,et al. Antibacterial activity of the aqueous extract of Thonningiasanguinea against Extended-Spectrum- $\beta$ - Lactamases (ESBL) producing Escherichia coli and Klebsiella pneumoniae strains. Tropical Journal of Pharmaceutical Research, September 2007; 6(3): 779-783. https://doi. org/10.4314/tjpr.v6i3.14659

20. MilosNikolic, Sava Vasic, JelenaDurdevic, OlgicaStefanovic and Ljiljanacomic. Antibacterial and Anti-biofilm activity of ginger (Zingiber officinale (roscoe)) ethanolic extract: Kragujevac J. Sci.2014; 36: 129-136. https://doi.org/10.5937/KgJSci1436129N

21. Anita Dua, Gaurav Garg, Sushil Nagar, Ritu Mahajan. Methanol extract of clove (Syzygium aromaticum Linn.) damages cells and inhibits growth of enteropathogens. Journal of Innovative Biology 2014; 1(4): 200-205.

22. Neelima Christopher, Rajesh Ramachandran and KhaleelAhamedThaha. Anti Biofilm and Anti Plasmid Activites of Syzygium aromaticum and Kaempheria galanga against $P$ seudomonas aeruginosa. Int.J.Curr. Microbiol.App.Sci. 2017; 6(1): 859-865. https://doi. org/10.20546/ijcmas.2017.601.101

23. Iram Liaqat, Najma Arshad, Muhammad Arshad, Safdar Ali Mirza, Nazish Mazhar Ali and AmmaraShoukat. Antimicrobial Activity of Some Medicinal Plants Extracts Against Food Industry Isolates. Pakistan J. Zool. 2017; 49(2), 523-530. https://doi.org/10.17582/ journal.pjz/2017.49.2.523.530

24. Dinesh Maharjan, Anjana Singh, Binod Lekhak, Shaila Basnyat, Lekhnath S. Gautam. Study on Antibacterial Activity of Common Spices. Nepal Journal of Science and Technology 2011; 12: 312-317. https://doi. org/10.3126/njst.v12i0.6518

25. N. S. Chavan, R. D. Phadtare, and T. B. Chavan. Effect of aqueous extracts of different medicinal plants on control of Streptococcus mutans. Int. J. Curr. Microbiol. App. Sci 2015; 4(4): 1072-1081.

26. I Liaqat, Q Pervaiz, S Jamil Bukhsh, SI Ahmed and N Jahan. Investigation of Bactericidal Effects of Medicinal Plant Extracts on Clinical Isolates and Monitoring Their Biofilm Forming Potential. Pak Vet J., 2016; 36(2): 159164.

27. S. Archana and Jayanthi Abraham. Comparative analysis of antimicrobial activity of leaf extracts from fresh green tea, commercial green tea and black tea on pathogens. Journal of Applied Pharmaceutical Science 2011; 01(08): 149-152.

28. Barat Ali Fakheri, Samaneh Bagheri, Nafiseh Mahdi Nezhad. Comparison of Antimicrobial and Antioxidant Activities of Four Different Tea Extracts. Med Microbiol Infec Dis, 2015, 3 (3-4): 57-61.

29. Zeinab Mohsenipour, Mehdi Hassanshahian. The Effects of Allium sativum Extracts on Biofilm Formation and Activities of Six Pathogenic Bacteria. Jundishapur J Microbiol. 2015; 8(8): e18971. https:// doi.org/10.5812/jjm.18971v2

30. Packia Lekshmi N. C. J., Viveka S., Jeeva S. and Raja Brindha J. Efficacy of crude extracts of Allium sativum and Allium cepa against human pathogens. Adv. Appl. 
Sci. Res. 2015; 6(1):72-78.

31. Shams S., Mehdipour A., Kermani S., Ghorbani H., Ragolia S., Anti-Biofilm Activity of Punica granatum, Ricinus communis, and Allium sativum Plant Extracts on Streptococcus mutans. Infection Epidemiology and Microbiology. 2018; 4(2):67-72.

32. Shatha Al Bayati. Antibacterial Effect of Ethanolic Extract of Allium sativum on Biofilm Forming Staphylococcus aureus which Cause Folliculitis. Int. J. Curr. Microbiol.App.Sci. 2018;7(01): 1904-1913. https://doi.org/10.20546/ijcmas.2018.701.229

33. V. Rathabai and Kanimozhi. D. Evaluation of Anti Microbial Activity of Coriandrum sativum. International Journal of Scientific Research and Reviews 2012; 1(3), 01-10.

34. Amel O. Bakhiet,Sabna D. Mohammed, Samia, M.A. El Badwi , Warda, S. Abdel Gadir, Abdel-GadirH.Alkhatim and S.E.I. Adam. Antimicrobial Activity of Petroselinum sativum and Coriandrum sativum Seeds. Research Journal of Microbiology 2006; 1: 346-352. https://doi. org/10.3923/jm.2006.346.352

35. Vasiee Alireza, Tabatabaei Yazdi Farideh, Mortazavi Seyed Ali. The Antibacterial Activity of Coriandrum (Coriandrum sativum) On Pathogenic Microorganisms "In vitro". Iranian Journal of Infectious Diseases And Tropical Medicine 2016; 20 (71): 59-66.

36. Bezalwar P. M, Shuddhalwar P. P. A Quest of Anti-Biofilm Activity of Zingiber officinale Root and Coriandrum sativum Seed Extract Against Clinical Isolates of Pseudomonas aeruginosa and Staphylococcus aureus. International Journal of Science and Research 2015; 4(4): 2581-84.

37. Karlapudi P. Abraham et al. Investigation of the Potential Antibiofilm Activities of Plant Extracts. Int J Pharm PharmSci., 2012; 4 (4): 282-285. 